\title{
The Left Hemisphere's Role in Hypothesis Formation
}

\author{
George Wolford, Michael B. Miller, and Michael Gazzaniga \\ Psychology Department, Dartmouth College, Hanover, New Hampshire 03755
}

In a probability guessing experiment, subjects try to guess which of two events will occur next. Humans tend to match the frequency of previous occurrences in their guesses. Animals other than humans tend to maximize or always choose the option that has occurred the most frequently in the past. Investigators have argued that frequency matching results from the attempt of humans to find patterns in sequences of events even when told the sequences are random. There is independent evidence that the left hemisphere of humans houses a cognitive mechanism that tries to make sense of past occurrences. We performed a probability guessing experiment with two splitbrain patients and found that they approximated frequency matching in their left hemispheres and approached maximizing in their right hemispheres. We obtained a conceptual replication of that finding on patients with unilateral damage to either the left or right hemisphere. We conclude that the neural processes responsible for searching for patterns in events are housed in the left hemisphere.

Key words: split-brain patients; interpreter; decision making; probability matching; maximizing; hypothesis generation
From our own experience and from experimental evidence, we know that people are prone to search for and posit causal relationships among events. One's relative or friend might remark that she cannot take vitamin $\mathrm{X}$ because it causes a rash. Such relationships are often made on the basis of scant data and are often false. Yellott (1969) provided a particularly striking demonstration of the extent to which people posit such causal relationships. In his experiment, a light was flashed to either the left or the right on each trial. Before each trial, subjects had to predict which of two lights would appear. Subjects participated in many trials in which the two lights appeared randomly with a probability that varied across blocks. In most blocks of trials, the most frequent light appeared with a $p=0.8$. Subjects' predictions matched the frequency of the actual presentations (frequency matching). In the final block, the experiment changed without the subjects' knowledge. At that point, the light appeared wherever the subject predicted it would. In other words, if the subject predicted the light would appear on the right, it did. If the subject predicted the light would appear on the left, it did. After 50 trials of this, Yellott stopped the experiment and asked subjects for their impressions. Subjects continued to predict the previously most frequent light $80 \%$ of the time during those last 50 trials but overwhelmingly responded that there was a fixed pattern to the light sequences and that they had finally figured it out. They proceeded to describe elaborate and complex sequences of right and left choices that resulted in their responses always being correct. This outcome supports the contention that subjects had been searching for causal sequences all along and were fooled into thinking they had succeeded.

In a variety of such guessing experiments, humans typically exhibit frequency matching. That is if the lights to the two sides are presented with probabilities $p$ and $(1-p)$, the subjects guess

Received Sept. 7, 1999; revised Jan. 3, 2000; accepted Jan. 5, 2000.

This work was supported by National Institute of Neurological Disease Grants NS 17778-16 and NS 31443-07 and the McDonnell Pew Foundations.

Correspondence should be addressed to George Wolford, Psychology Department, Dartmouth College, 6207 Gerry Hall, Hanover, NH 03755. E-mail: george. wolford@dartmouth.edu.

Copyright (C) 2000 Society for Neuroscience $\quad 0270-6474 / 00 / 200001-\bullet \$ 15.00 / 0$ the two lights with probabilities $p$ and $(1-p)$ (Humphreys, 1939; Estes, 1961). The tendency to match frequency has intrigued investigators because it is a nonoptimal strategy for this paradigm. Maximizing, or choosing the most frequent option all of the time, yields more correct guesses than matching as long as $p \neq$ 0.5 . In other words, if the red light occurs with a frequency of $70 \%$ and a green light occurs with a frequency of $30 \%$, overall accuracy will be highest if the subject predicts red all of the time (maximizing). Frequency matching will lead to correct answers $58 \%$ of the time $(0.7 * 0.7+0.3 * 0.3)$. Maximizing will lead to correct answers $70 \%$ of the time $(0.7 * 1.0+0.3 * 0.0)$. Interestingly, most other animals maximize in such paradigms (Hinson and Staddon, 1983). So why do humans choose a less optimal strategy than rats? Our view is that humans believe there is a pattern, even if told the sequence is random, and they attempt to figure out the pattern. Any reasonable pattern hypothesized by the subjects would have to match frequency if it were to be a correct hypothesis. Perhaps animals other than humans adopt a more optimal strategy than humans in this paradigm, because they are not as hindered by the tendency to search for and posit causal hypotheses.

Gazzaniga (1989, 1995) and Metcalfe et al. (1995) have hypothesized the existence of an interpreter that plays the role of trying to make sense out of the information that it confronts, in other words, generating causal hypotheses. Using split-brain patients, Gazzaniga (1995) provided evidence that this interpreter is located in the left hemisphere in most individuals. The simulta-

This article is published in The Journal of Neuroscience, Rapid Communications Section, which publishes brief, peerreviewed papers online, not in print. Rapid Communications are posted online approximately one month earlier than they would appear if printed. They are listed in the Table of Contents of the next open issue of JNeurosci. Cite this article as: JNeurosci, 2000, 20:RC64 (1-4). The publication date is the date of posting online at www.jneurosci.org.

http://www.jneurosci.org/cgi/content/full/4017 
Figure 1. Probability guessing behavior in each hemisphere of a split-brain patient (J.W.) relative to the past frequency of presentation. Error bars represent SD based on the assumption of Bernoulli trials.

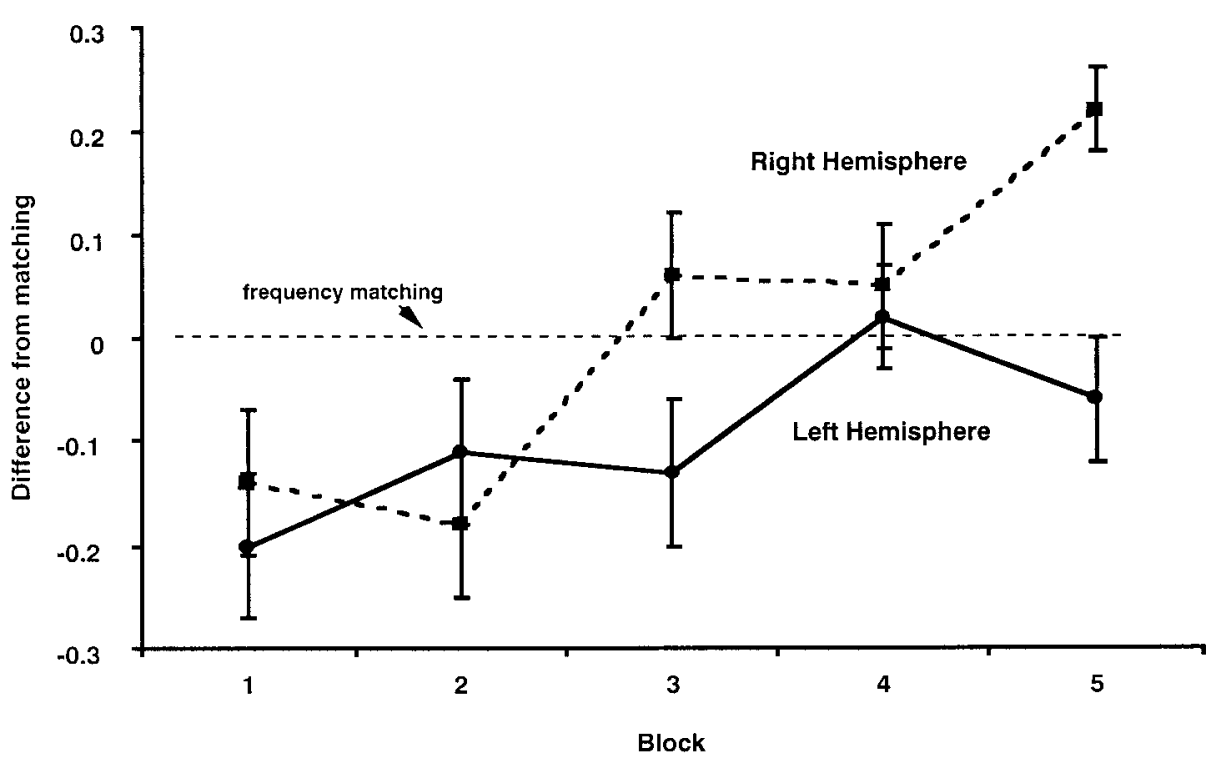

neous concept test provides an example of the function of the interpreter. In this task, a split-brain patient is shown a picture exclusively to the left hemisphere (e.g., a chicken) and another picture exclusively to the right hemisphere (e.g., a snow scene). The patient is then given an array of pictures and asked to point to a picture associated with the presented pictures. In the above example, the left hemisphere chose a chicken claw, and the right hemisphere chose a shovel. When asked to explain the choices, the patient responded, "Oh, that's simple. The chicken claw goes with the chicken, and you need a shovel to clean out the chicken shed." The right hemisphere is unable to produce speech, so it cannot explain its selection. The left hemisphere is unaware of the picture that the right hemisphere is responding to (i.e., the snow scene), so it must generate its own interpretation of why the left hand pointed to a shovel. The left hemisphere, observing the actions of the left hand and right brain, interprets those actions within the context of what it knows (i.e., a chicken claw) and generates an explanation for the shovel that is consistent with its knowledge (Gazzaniga, 1989).

\section{EXPERIMENT 1}

We hypothesized that the interpreter might be the structure that underlies the tendency to posit causal explanations and may be responsible for the frequency matching observed in probability guessing experiments. To test this, we presented separate probability guessing experiments to the two hemispheres of two splitbrain patients, predicting frequency matching in the left hemisphere and maximizing in the right.

\section{Materials and Methods}

J.W. and V.P. were split-brain patients whose corpus callosi had been severed as treatment for epilepsy. Each participated in five blocks of 100 trials each. All stimuli were presented and all responses were collected on a computer. Each trial began with a row of three arrows $(>>>)$ pointing right or left. The arrows signified which visual field to make a prediction in. If the arrows pointed to the right, they were told that either a small green square would be presented toward the top of the computer screen on the right side or a small red square would be presented toward the bottom of the screen on the right side. They were instructed to guess whether the square would be at the top or the bottom by pressing the appropriate key on the right side of the keyboard with their right hand. One hundred milliseconds after their guess, a square was presented $4^{\circ}$ to the right of fixation for $100 \mathrm{msec}$. The top square was presented with $p=$ 0.8 , and the bottom square was presented with $p=0.2$. If the arrows were pointing to the left, everything above was reversed. They were instructed to guess whether a top square or bottom square would be presented in the left visual field. The probability of a top square in the left visual field was $p=0.7$, and the probability of a bottom square was $p=0.3$. The sequence in the left visual field was independent of the sequence in the right visual field. All of the sequences were generated randomly using the random number generator in the computer language. Both subjects were told to always maintain fixation on the center arrows. V.P.'s eye position was monitored with an ISCAN tracking system. Feedback on the proportion of correct guesses was provided at the end of each block of trails.

\section{Results}

The results of the first experiment are shown in Figures 1 and 2. The error bars are based on the assumption of Bernoulli trials. As predicted, when J.W. and V.P. made guesses about stimuli presented to the left hemisphere, they came close to matching the frequency of occurrence of previous stimuli (V.P. undershot frequency matching slightly). When they made guesses about stimuli presented to the right hemisphere, they moved steadily toward maximizing, and both were choosing the more frequent square $>20 \%$ over frequency matching by the final block. As an alternative measure of performance, we calculated the value of criterion from signal detection theory. Taking the most frequent alternative as the signal, one would expect criterion to be more liberal as performance approaches maximizing. For J.W., the criterion in the final block was estimated to be -1.38 in the right hemisphere with a $95 \%$ confidence interval of \pm 0.065 . His criterion in the left hemisphere was $-0.651 \pm 0.036$. Negative values indicate a more liberal criterion, so the criterion was substantially more liberal in the right hemisphere. For V.P. the criterion in the right hemisphere was $-1.83 \pm 0.257$ and in the left hemisphere was $-0.31 \pm 0.033$. Again, V.P.'s criterion was more liberal in the right hemisphere. The difference between the hemispheres is not evident immediately but emerges over trials. The likely reasons for this are presented in Discussion.

\section{EXPERIMENT 2}

As a conceptual replication to the finding with the split-brain patients, we carried out a similar paradigm on a series of patients with unilateral damage to the frontal and prefrontal cortex. Be- 

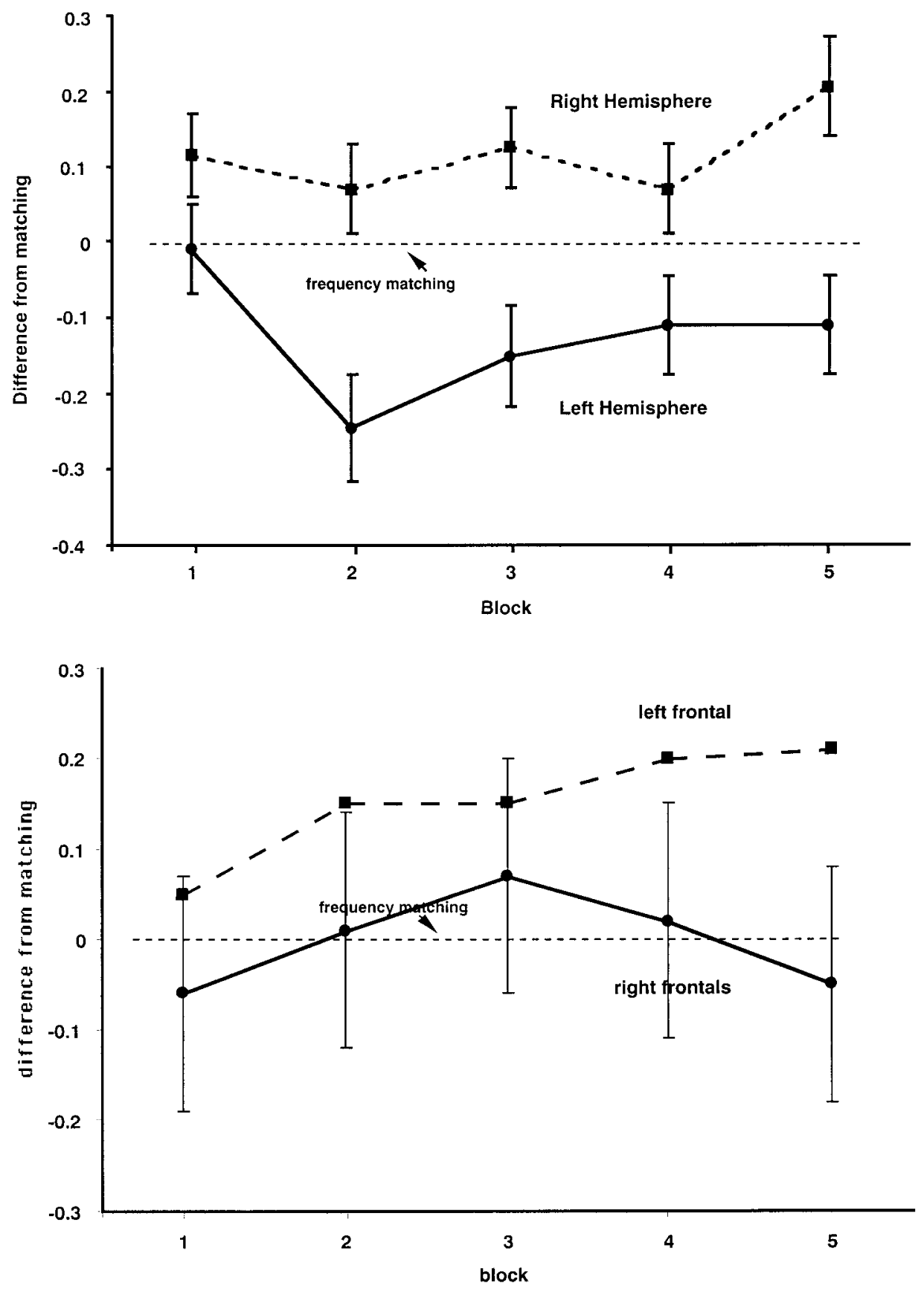

Figure 2. Probability guessing behavior in each hemisphere of a split-brain patient (V.P.) relative to the past frequency of presentation. Error bars indicate SD based on the assumption of Bernoulli trials.

Figure 3. Probability guessing behavior in four patients with unilateral right hemisphere damage and one patient with unilateral left hemisphere damage relative to the past frequency of presentation. Error bars represent $\pm 2 \mathrm{SD}$ around the mean of the right frontal patients in each block.

cause the interpreter is assumed to be localized in the frontal and prefrontal areas of the left hemisphere, we predicted that the patient with damage to that area would show maximizing as in the right hemisphere of the split-brain patient. The patients with unilateral damage to the right hemisphere should have an intact interpreter and were predicted to show frequency matching.

\section{Materials and Methods}

Five patients participated in a conceptual replication. All five patients had unilateral, focal lesions to the dorsolateral prefrontal cortex as a result of stokes, as revealed by high-resolution magnetic resonance imaging scans (for details on patients' lesions, see Swick and Knight, 1996). Four of the patients had lesions localized to the right prefrontal cortex, and one patient had a lesion localized to the left prefrontal cortex. The ages of the patients ranged from 33 to 79 years old. The one left frontal patient was nonaphasic. The procedure for these patients was identical in every respect to the procedure used in Experiment 1.

\section{Results}

The results of the replication are shown in Figure 3. The error bars in Figure 3 represent $\pm 2 \mathrm{SD}$ around the mean of the right frontal patients in each block. As predicted, the four patients with right frontal damage exhibited choices close to frequency matching, whereas the single left frontal patient was significantly closer to maximizing. The effect was fairly clear, because the left frontal patient was closer to maximizing in every block than any right frontal patient.

\section{DISCUSSION}

The results from both split-brain patients and from the patients with unilateral damage to the frontal cortex show approximate frequency matching in the left hemisphere and movement toward maximizing in the right hemisphere. The right hemisphere data 
closely mirror data produced by animals other than humans. We analyzed the data in terms of probability relative to frequency matching. Nearly identical patterns are revealed when using absolute probabilities. In both experiments, the difference between the hemispheres emerges over trials and is most evident in the final blocks. This is not surprising, because either strategy requires information about the nature of the sequences, and it requires some number of trials to extract that information. The situation is complicated for the two split-brain patients, because they experience two simultaneous and independent sequences, one in the left field and one in the right. Furthermore, both are patients with a history of epilepsy and brain surgery. The frontal patients have experienced major brain traumas and function reasonably well but with some loss of cognitive functioning. In both the previous human and nonhuman animal literature, frequency matching or maximizing emerges over trials in a relatively gradual fashion.

Why might we try to search for and posit these causal relationships? Clearly, it would be of great utility to search for causal relationships among events if such relationships existed. From an evolutionary perspective, finding such relationships may have had survival value. Those who could uncover simple causal relationships such as determining which caves would stay dry and where the game could be found might live longer and produce more offspring. Although this tendency to search for causal relationships has potential benefits, it can lead to nonoptimal behavior when there is no simple causal relationship. Some of the common errors in decision making are consistent with the notion that we are prone to search for and posit causal relationships even when the evidence is insufficient or even random. We find that the search for causal explanations appears to be a left hemisphere activity, consistent with previous research on the interpreter.

\section{REFERENCES}

Estes WK (1961) A descriptive approach to the dynamics of choice behavior. Behav Sci 6:177-184.

Gazzaniga MS (1989) Organization of the human brain. Science 245:947-952.

Gazzaniga MS (1995) Principles of human brain organization derived from split-brain studies. Neuron 14:217-228.

Hinson JM, Staddon JER (1983) Matching, maximizing and hillclimbing. J Exp Anal Behav 40:321-331.

Humphreys LG (1939) Acquisition and extinction of verbal expectations in a situation analogous to conditioning. J Exp Psychol 25:294-301.

Metcalfe J, Funnell M, Gazzaniga MS (1995) Right-hemisphere superiority: studies of a split-brain patient. Psychol Sci 6:157-163.

Swick D, Knight RT (1996) Is prefrontal cortex involved in cued recall? A neuropsychological test of PET findings. Neuropsychologica 34:1019-1028.

Yellott Jr JI (1969) Probability learning with noncontingent success. J Math Psychol 6:541-575. 